Article

\title{
Sensitivity Improvement of Quantum Dot-Blended Hybrid Detector for X-ray Imaging
}

\author{
Seahong Kim, Jehoon Lee $®$ and Jungwon Kang * \\ Department of Electronic and Electrical Engineering, Dankook University, Gyenggi-do 16890, Korea; \\ honge9210@naver.com (S.K.); usyj0512@gmail.com (J.L.) \\ * Correspondence: jkang@dankook.ac.kr; Tel.: +82-031-8005-3646
}

Received: 10 January 2020; Accepted: 25 February 2020; Published: 1 March 2020

check for updates

\begin{abstract}
This study investigated the characteristics of an indirect-type hybrid X-ray detector with a conjugated polymer poly(3-hexylthiophene) (P3HT) and CdSe quantum dot (QD) blended active layer. To improve detection sensitivity, the optimal blending ratio of P3HT:CdSe QDs, ligand exchange effect, and optimal process condition of the active layer were examined. The detector with a P3HT:CdSe QDs $=1: 5$ blended active layer showed the highest collected charge density (CCD) and highest sensitivity under X-ray irradiation. The replacement of a trioctylphosphine (TOP) ligand by a pyridine ligand effectively assisted the charge transport and reduced the QD aggregation, increasing the detection sensitivity of the detector by $75 \%$ after the ligand exchange. To further improve the sensitivity of the proposed detector, the optimized process conditions of the active layer were studied. The sensitivity of the detector with an active layer of about $80 \mathrm{~nm}$ thickness formed by a double-coating method showed the highest CCD of $62.5 \mathrm{nA} / \mathrm{cm}^{2}$, and the highest sensitivity of $0.14 \mathrm{~mA} / \mathrm{Gy} \cdot \mathrm{cm}^{2}$. Due to additional pyridine treatment between the double-coating processes, the surface roughness of the active layer decreased, and the CCD and sensitivity subsequently increased.
\end{abstract}

Keywords: Quantum dots; hybrid detector; indirect X-ray detector; ligand exchange

\section{Introduction}

Organic semiconductor materials offer advantages such as low manufacturing cost, mechanical flexibility, light weight, and excellent absorbance. Inorganic nanoparticles (NPs) in the form of quantum dots (QDs), nanowires, and nanoplatelets (NPLs) have the advantages of high quantum efficiency, high carrier mobility, and excellent stability. Therefore, photoelectric devices with both inorganic and organic materials applied together may combine the advantages of both materials. Organic-inorganic nanocrystalline structures are complex but have attracted interest in scientific and industrial fields. Research has been carried out on hybrid photovoltaic devices containing NPs, such as $\mathrm{Au}$ [1], $\mathrm{TiO}_{2}$ [2], and $\mathrm{ZnO}$ [3], and QDs, such as CdSe [4], PbS [5], CdS [6], and CdTe [7]. Recently, research on hybrid $X$-ray detectors using both inorganic and organic materials has been conducted. High atomic number (high-Z) NPs, such as $\mathrm{Bi}_{2} \mathrm{O}_{3}[8,9]$, QDs, such as CdSe [10] and PbS [11], and scintillating NPs, such as terbium-doped gadolinium oxysulfide (GOS:Tb) [12], were applied to the hybrid detector. Metal and metal oxide NPs show good chemical and physical stability, good carrier mobility, and efficient photon absorption at the organic polymer interface. In addition, QDs have the advantages of tunable bandgap and excellent quantum efficiency. Therefore, both NPs and QDs create additional percolation paths to the carriers to facilitate current collection, and they enhance X-ray absorption and visible-light absorption to promote exciton generation.

There are two X-ray detection methods: direct $[13,14]$ and indirect $[15,16]$. In direct detection, the incident $\mathrm{X}$-rays are directly converted to charges in the photoconductor layer of the detector, whereas in scintillator-coupled indirect detection, the incident $X$-rays are converted into visible light 
through the scintillator, and visible light is absorbed into the active layer of the detector to generate charges. In this study, we investigated a hybrid-type indirect detector with an active layer mixed with organic materials and QDs to improve X-ray conversion efficiency. The active layer was composed of conjugated polymer poly(3-hexylthiophene) (P3HT) as the electron donor and $5 \mathrm{~nm}$ CdSe QDs as the electron acceptor. The P3HT absorbs visible photons and produces excitons. Blending QDs with the conjugated polymers improves the absorbance and charge collection. Figure 1a shows a schematic of the detector, while Figure $1 \mathrm{~b}$ depicts the corresponding energy band diagram of the detector. The active layer was composed of P3HT and CdSe QDs. The CsI(Tl) scintillator was coupled with the detector to convert incident $X$-ray photons to visible-light photons. The lowest unoccupied molecular orbital (LUMO) and the highest measured occupied molecular orbital (HOMO) levels of CdSe QD acceptors are closer to each of the levels of the P3HT donor. Therefore, the carriers generated at the donor/acceptor interfaces can be easily transferred. In order to improve the detection performance according to the P3HT-QDs blending and processing conditions, the radiation detector parameters, such as the sensitivity, collected charge density (CCD), and dark current density (DCD), were measured under X-ray exposure. To understand the characteristic of the detector without the scintillator, the short-circuit current density (JSC), series resistance $\left(\mathrm{R}_{\mathrm{S}}\right)$, and power conversion efficiency (PCE) were measured under artificial solar illumination. We also evaluated the ligand exchange effect of CdSe QDs on detector performance affecting the electrical properties and surface morphology [17-20].

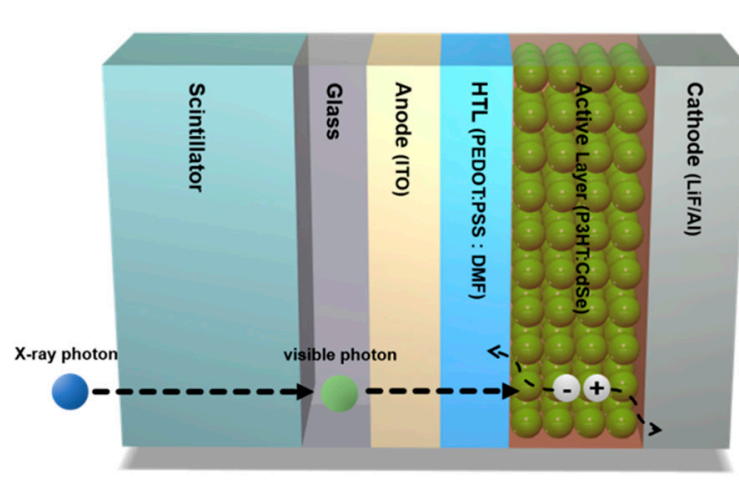

(a)

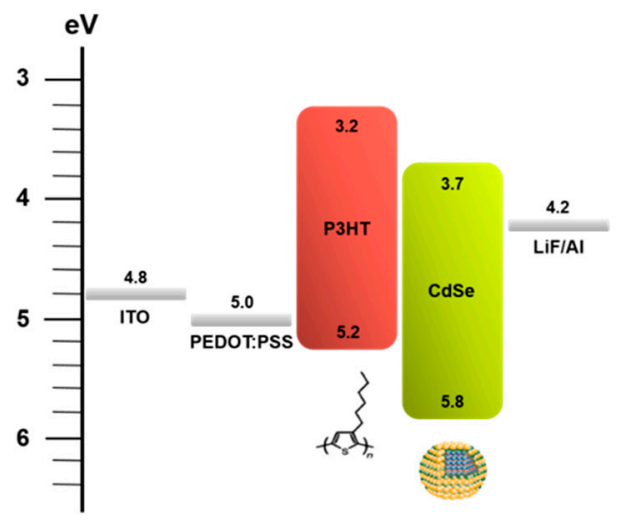

(b)

Figure 1. (a) Schematic, and (b) corresponding energy band diagram of the proposed X-ray detector with P3HT:CdSe active layer.

\section{Experiment Details}

\subsection{Hybrid Detector Fabrication}

Figure 2a shows the sequence to fabricate the hybrid detector with a P3HT:CdSe active layer. After patterning an indium tin oxide (ITO) electrode applied as the anode, the ITO-patterned glass substrate was cleaned sequentially by ultrasonic treatment in acetone, methanol, and isopropyl alcohol. A poly(3,4-ethylenedioxythiophene):poly(styrenesulfonate) (PEDOT:PSS) solution mixed with $50 \mathrm{wt} . \%$ dimethylformamide (DMF) was spin-coated onto the ITO anode at $3000 \mathrm{rpm}$, then baked on a hot plate at $160{ }^{\circ} \mathrm{C}$ for $30 \mathrm{~min}$ under air atmosphere. The thickness of the PEDOT:PSS layer was about $30 \mathrm{~nm}$. The PEDOT:PSS layer was applied to improve the hole transport and flatness of the ITO-patterned surface. The CdSe QDs dissolved in toluene were refined with a micro-centrifuge to obtain the pristine CdSe QDs. In general, the QDs showed a very uniform size distribution. The mean diameter of QDs used in this experiment was $3.6 \mathrm{~nm}$ and standard deviation was $1.3 \mathrm{~nm}$. Instead of mean diameters, D90 values are commonly used in particle size analysis. In our case, $90 \%$ of the QDs were smaller than $5.5 \mathrm{~nm}$ (D90 value), and we defined them as $5 \mathrm{~nm}$ diameter QDs. The $5 \mathrm{~nm}$ diameter QDs were selected among various QDs sizes in consideration of the absorbance and uniform dispersion characteristics 
obtained through the study of the organic X-ray detector with the additive QDs [10]. To make the P3HT:CdSe QD solution, P3HT and CdSe QD were dissolved in $1 \mathrm{~mL}$ of toluene with the different weight ratios of 1:2, 1:3, 1:4, 1:5, and 1:6. The weight of the P3HT polymer dissolved in the P3HT:CdSe QD solution was fixed at $7.5 \mathrm{mg}$. Therefore, the weight of CdSe QDs was increased to $45 \mathrm{mg}$ at the blending condition of P3HT:CdSe QD = 1:6. The P3HT:CdSe QD solution was stirred at $70{ }^{\circ} \mathrm{C}$ for 3 $\mathrm{h}$, and then spin-coated onto the PEDOT:PSS layer at $1100 \mathrm{rpm}$. The baking condition was $150{ }^{\circ} \mathrm{C}$ for $10 \mathrm{~min}$ under air atmosphere. The cathode, consisting of $0.5 \mathrm{~nm}$ of $\mathrm{LiF}$ and $120 \mathrm{~nm}$ of $\mathrm{Al}$, was deposited on the active layer by thermal evaporation under a pressure of $10^{-7}$ torr. The detector was encapsulated with a cover-glass to prevent oxygen and humidity penetration. Figure $2 b$ shows the fabricated detector, and four cells with an active area of $4 \mathrm{~mm}^{2}$ formed on the detector.

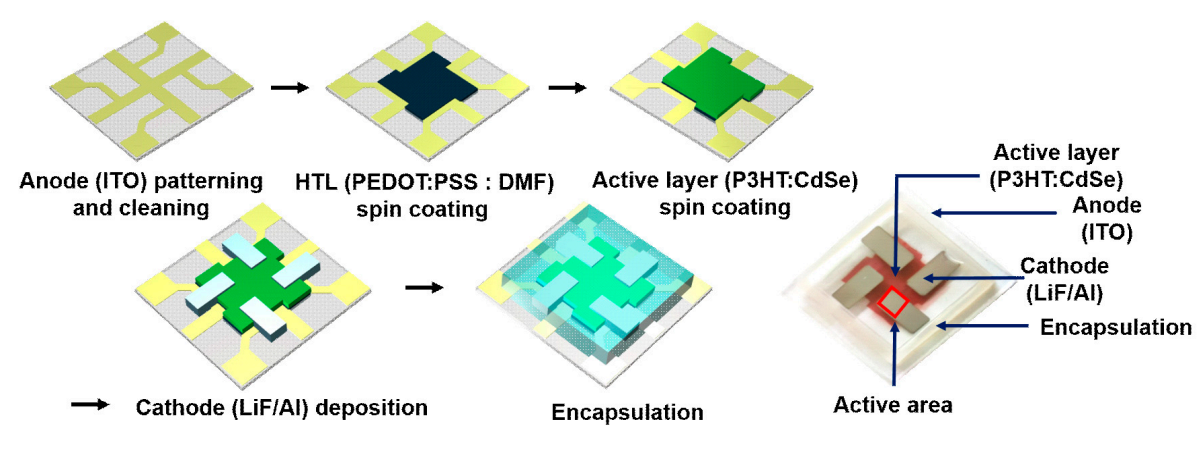

(a)

(b)

Figure 2. (a) Process flow of the hybrid detector with the P3HT:CdSe quantum dots (QDs) active layer, and (b) the actual image of the fabricated detector.

\subsection{Experimental Set-Up}

Figure 3 shows the experimental set-up to evaluate the properties of the X-ray detector. The set-up consisted of an X-ray generator (AJEX 2000H), a solar simulator (San Ei Elec. XES-40S2-CE), and an electrometer (Keithley 6517B) for the measurement of current density-voltage $(J-V)$ characteristics. The detector was evaluated in two ways: one was assessment after separating a scintillator from the detector, while the other was after combining with a scintillator. Prior to the experiment with the X-ray generator, the detector without the scintillator was exposed to the light of an AM 1.5 G filtered Xe lamp in a solar simulator. Bias from -1.0 to $1.0 \mathrm{~V}$ was applied to the detector for the collection of generated charges. Among the various parameters obtained during artificial solar irradiation, the short-circuit current density (JSC), power conversion efficiency (PCE), and series resistance $\left(\mathrm{R}_{\mathrm{S}}\right)$ were calculated from the $J-V$ characteristics of the detector. By analyzing these parameters, the inherent property of the detector without the scintillator could be easily estimated.

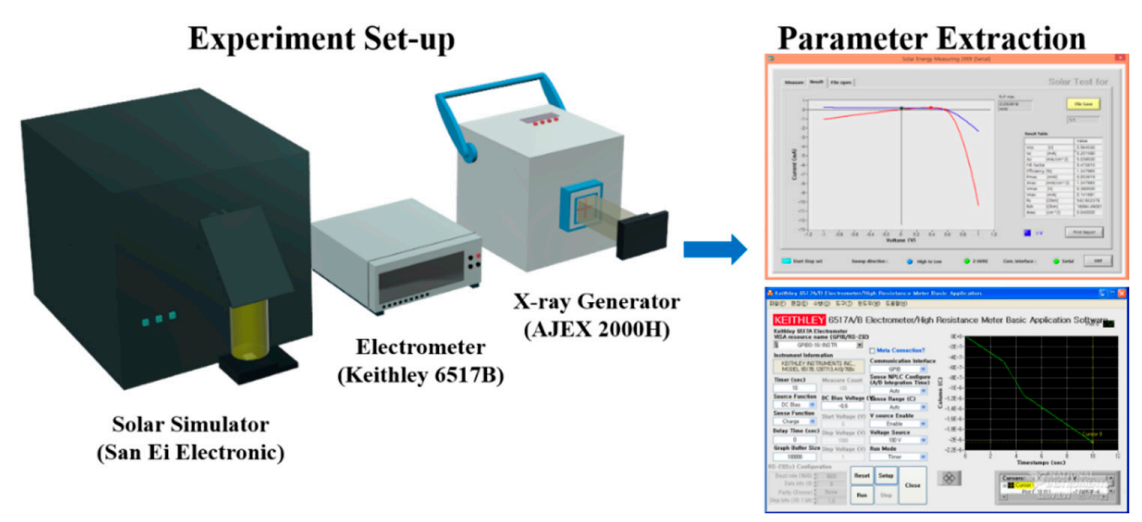

Figure 3. Experimental set-up for measuring the solar parameters $\left(\mathrm{J}_{\mathrm{sc}}, \mathrm{PCE}\right.$, and $\left.\mathrm{R}_{\mathrm{s}}\right)$ and $\mathrm{X}$-ray parameters (CCD, DCD, and sensitivity). 
The photoelectric characteristics of the detector with the scintillator (Hamamatsu Photonics J1311), consisting of $0.5 \mathrm{~mm}$ of $\mathrm{Al}$ and $0.4 \mathrm{~mm}$ of $\mathrm{CsI}(\mathrm{Tl})$, were evaluated under X-ray irradiation. For all experiments, the operation condition of the $\mathrm{X}$-ray generator was fixed at $80 \mathrm{kV}_{\mathrm{p}}$ and $60 \mathrm{mAs}$. The exposure time was also fixed at $1.57 \mathrm{~s}$. For the collection of generated charges, bias from 0 to $1.0 \mathrm{~V}$ was applied to the detector. The collected current density (CCD) during X-ray irradiation was calculated using Equation (1), while the dark current density (DCD) on non-irradiation of X-ray was calculated using Equation (2):

$$
\begin{aligned}
& C C D\left[\frac{n A}{c m^{2}}\right]=\frac{\text { CollectedChargeduring } X-\text { rayON }}{\text { ExposedTime } \times \text { ExposedDetectionArea }} \\
& D C D\left[\frac{n A}{\mathrm{~cm}^{2}}\right]=\frac{\text { CollectedChargeduring } X-\text { rayOFF }}{\text { ExposedTime } \times \text { ExposedDetectionArea }}
\end{aligned}
$$

The sensitivity, which represents the amount of generated charges in proportion to the absorbed dose, is defined in Equation (3). The absorbed X-ray dose was converted from the exposure, which was measured using the ion chamber (Capintec CII50) in the same position as the detector:

$$
\text { Sensitivity }\left[\frac{m A}{G y \cdot \mathrm{cm}^{2}}\right]=\frac{C C D \text { during } X-\text { rayON }-D C D \text { during } X-\text { rayOFF }}{\text { AbsorbedDose }}
$$

\section{Results and Discussion}

First, the characteristics of the indirect-type hybrid X-ray detector were examined according to the change in CdSe QD blending conditions. Figure 4a shows the absorption spectrum for the P3HT:CdSe (weight ratios of 1:2, 1:3, 1:4, 1:5, and 1:6) thin films, emission spectra for the AM 1.5 G filtered Xe lamp, and emission spectra for the CsI(Tl) scintillator under X-ray irradiation. As the content of CdSe QDs constituting the active layer increased, the absorbance tended to increase due to its high extinction coefficient. The highest absorbance was obtained at the condition of P3HT:CdSe QDs = 1:6. The emission spectrum of the CsI(Tl) scintillator showed its maximum peak at $560 \mathrm{~nm}$ and was well-matched with the absorption spectrum of the P3HT:CdSe QD blended film. The $J-V$ characteristics of the scintillator-decoupled detector were measured using the solar simulator. Figure $4 \mathrm{~b}$ shows the short-circuit current density (JSC) at the bias of $0 \mathrm{~V}$, power conversion efficiency (PCE) related to the photon-to-charge conversion efficiency, and series resistance $\left(R_{S}\right)$ of the detector calculated from the $J-V$ curves. The $\mathrm{J}_{\mathrm{SC}}$ and PCE increased as the P3HT:CdSe QD blending ratio changed from 1:2 to 1:5. The highest $\mathrm{J}_{\mathrm{SC}}$ and PCE were found at P3HT:CdSe QDs = 1:5 blending ratio.
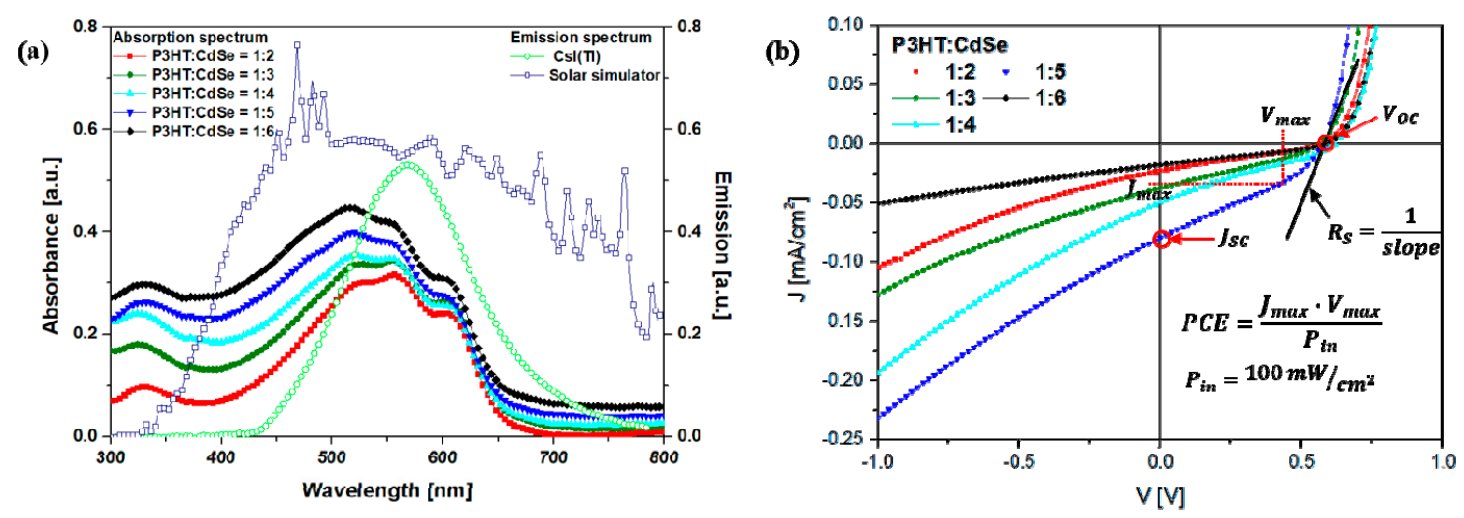

Figure 4. (a) The absorption spectrum for P3HT:CdSe QD thin films with different blending ratios of 1:2, 1:3, 1:4, 1:5, and 1:6 and emission spectra for CsI(Tl) scintillator under X-ray irradiation, and (b) $J-V$ characteristics of the proposed detectors with different blending ratios of P3HT:CdSe QDs obtained under artificial solar illumination. 
Figure 5a shows increasing the CdSe QDs content in the active layer tended to increase the JSC and PCE values associated with the detector's performance. When a large amount of QDs was blended in the active layer, the absorbance in the visible region was increased, and the visible light absorbed by the QDs was increased. As a result, CdSe QDs produced additional charges and were dispersed in P3HT to form an inter-network to improve charge transport and collection. The reason for the decrease in JSC and PCE at the conditions of P3HT:CdSe QDs $=1: 6$ is explained later.

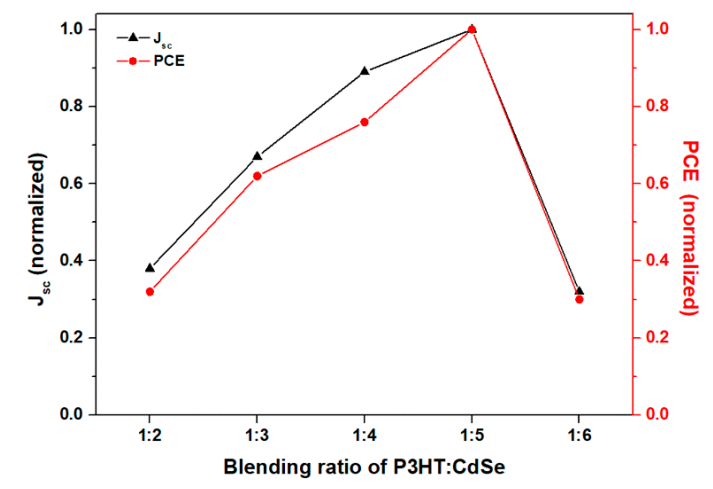

(a)

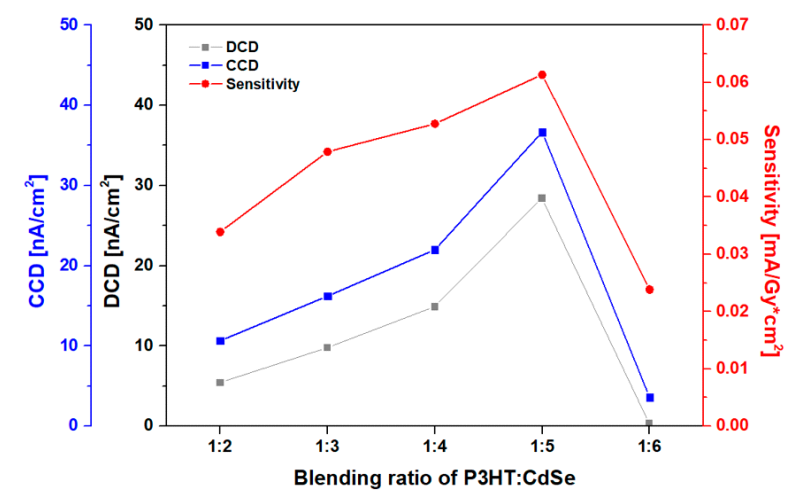

(b)

Figure 5. (a) The normalized solar parameters (JSC and PCE), and (b) radiation parameters (CCD, DCD, and sensitivity) of the detectors with different blending ratios of 1:2, 1:3, 1:4, 1:5, and 1:6 of the P3HT:CdSe QDs active layer.

To measure the amount of charge generated during X-ray irradiation, the detector was coupled with the $\mathrm{CsI}(\mathrm{Tl})$ scintillator. The operation condition for the $\mathrm{X}$-ray generator, located $30 \mathrm{~cm}$ away from the detector, was described in Section 2.2. Five different conditions of P3HT:CdSe QDs = 1:2, $1: 3,1: 4,1: 5$, and $1: 6$ of detectors were exposed to the $\mathrm{X}$-ray at $0.6 \mathrm{~V}$ bias. Equation (3) describes how the detector's sensitivity is determined by the difference between the CCD and DCD and is inversely proportional to the absorbed dose. The detector with the P3HT:CdSe QDs = 1:5 active layer showed the highest $C C D$ of $36.67 \mathrm{nA} / \mathrm{cm}^{2}$ and the highest sensitivity of $0.061 \mathrm{~mA} / \mathrm{Gy} \cdot \mathrm{cm}^{2}$. Figure $5 \mathrm{~b}$ shows the CCD and sensitivity obtained during X-ray radiation tended to be similar to the JSC and PCE obtained during solar illumination.

According to the AFM images in Figure 6, as the content of CdSe QDs in the active layer increased, the average roughness ( $\mathrm{Ra}$ ) of the active layer increased. Increasing the Ra value increased the surface area and effectively absorbed light. However, at the condition of P3HT:CdSe QDs = 1:6, the CdSe QDs tended to aggregate to form larger structures, which are similar to the solid bulk. This may interfere with charge transfer and inhibit exciton formation, which can reduce the conversion efficiency of photoelectric devices [21]. Therefore, the series resistance $\left(R_{S}\right)$ of the detector decreased with the increase of surface area until the condition of P3HT:CdSe QDs $=1: 5$, and then it rapidly increased at the condition of P3HT:CdSe QDs = 1:6. The expected quantum confinement effect on QDs also decreased, resulting in a decrease in exciton generation.

Ligand exchange in QDs was tested to improve the detector's sensitivity. Colloidal QDs are synthesized in liquid solutions, so passivating ligands are needed to avoid aggregation. Trioctylphosphine (TOP), which we applied, is often selected as a stabilizing ligand during synthesis. Charge transfer between neighboring QDs is often hindered by thick surfactant layers. Thus, one of the ways to improve charge transfer in photoelectric devices is replacing long ligand molecules with short ligand molecules [18]. Ligands affect not only the binding between QDs but also the surface traps and surface stability of QDs. Therefore, a ligand exchange experiment was conducted by selecting pyridine, one of the short ligands, instead of a TOP ligand. Figure 7 shows the ligand exchange sequence for CdSe QDs. The pyridine solution and CdSe QDs dissolved in toluene were mixed in a micro-tube at a volume ratio of 2:5. When the mixed solution was stirred overnight at $30^{\circ} \mathrm{C}$, the 
TOP ligand of CdSe QDs was exchanged with pyridine. To purify colloidal CdSe QDs, methanol was mixed with the CdSe QDs pyridine ligand solution in a volume ratio of 1:1. Sequential precipitations employing methanol as an anti-solvent were applied to obtain the QDs free from residual TOP ligands. The exchange process proceeded with binding the pyridine ligands in a solution to non-passivated $\mathrm{Cd}$ sites on the QD surface [22]. The purified CdSe QDs with pyridine ligand was obtained using a microcentrifuge method.

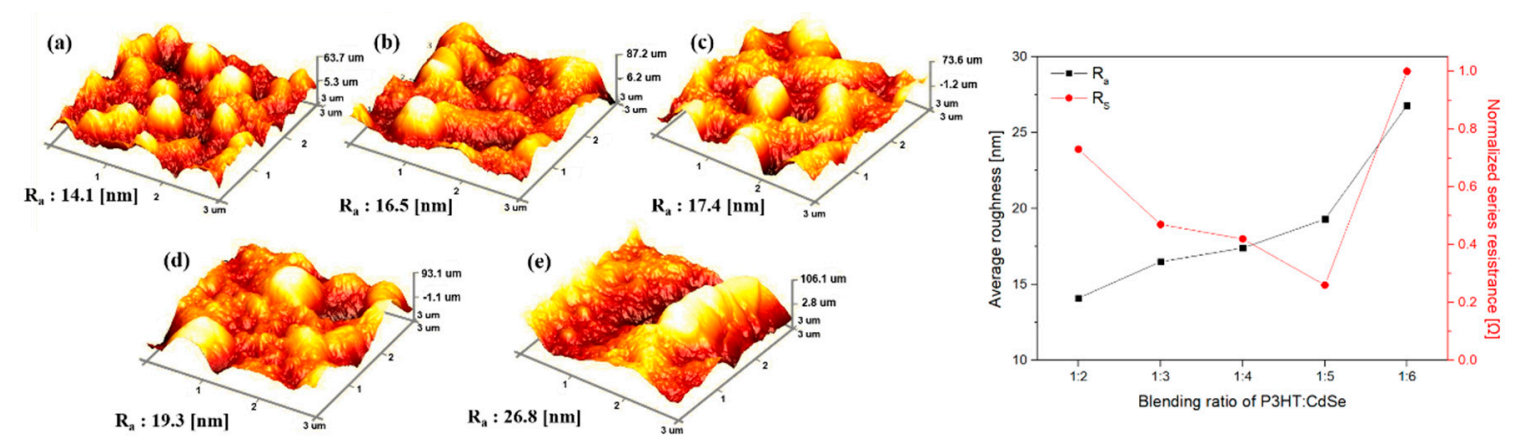

Figure 6. AFM images, average surface roughness (Ra) of the active layer under different blending ratios of P3HT:CdSe QDs of (a) 1:2, (b) 1:3, (c) 1:4, (d) 1:5, and (e) 1:6.

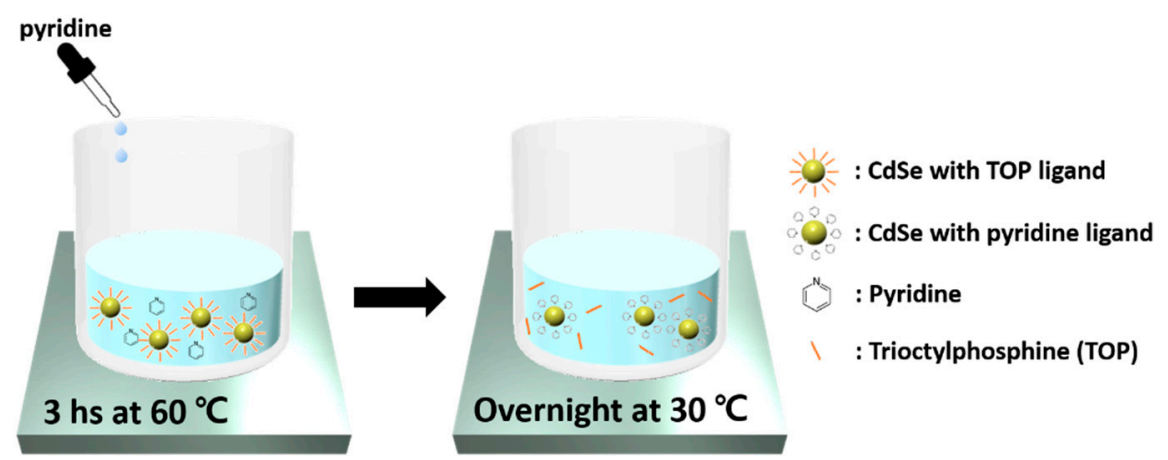

Figure 7. Ligand exchange sequence substituted with pyridine.

Ligand's effect on charge transfer was investigated by carrier mobility calculation based on ligand exchange. The optimized active layer condition (P3HT:CdSe QDs $=1: 5$ ) obtained from previous experiments was applied to the detector preparation. Carrier mobility was obtained using the space charge limiting current (SCLC) model from the $J-V$ characteristics in Figure 8a measured under dark conditions $[23,24]$. The SCLC model is useful for explaining the charge transport properties of single-crystal and polycrystalline organic thin films. Figure $8 \mathrm{~b}$ shows the SCLC region was defined as the region having a slope of 2 to 2.5 in the log scale graph. The mobility was calculated using the following Mott-Gurney equation in the SCLC domain:

$$
\mu=\frac{8}{9} \cdot J \cdot \frac{L^{3}}{V_{a}^{2} \cdot \varepsilon_{0} \cdot \varepsilon_{r}}
$$

where $J$ is the current density, $V_{a}$ is the applied voltage, $\varepsilon_{0}$ is the free-space permittivity, $\varepsilon_{r}$ is the relative permittivity of applied material, and $L$ is the thickness of the active layer, all of which values are listed in Table 1. The thickness of the active layer was measured using a surface profiler (KLA-Tencor Alpha-step AS-500). In Equation (4), the relative permittivity of the P3HT:CdSe QDs mixed material was determined using the permittivity equation of the mixed material [25], and the calculated relative permittivity also changed upon ligand exchange of CdSe QDs. The mobility in the detector with P3HT:CdSe QDs with TOP ligand was $8.33 \times 10^{-8} \mathrm{~cm}^{2} / \mathrm{V} \cdot \mathrm{s}$, and the mobility in the detector with 
P3HT:CdSe QDs with pyridine ligand was $1.03 \times 10^{-6} \mathrm{~cm}_{2} / \mathrm{V} \cdot \mathrm{s}$. Ligand exchange increased the mobility by more than 10 times.
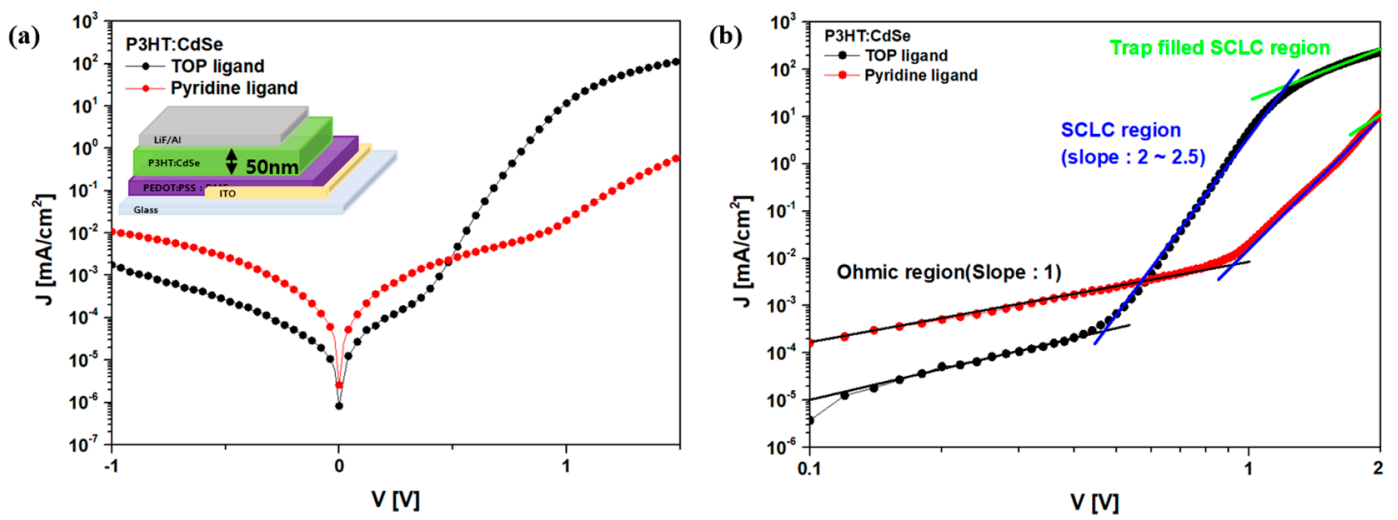

Figure 8. (a) $J-V$ characteristics of the detector with P3HT:CdSe QDs = 1:5 active layer measured under dark conditions (TOP and pyridine ligands applied), and (b) space charge limited current (SCLC) behavior of the detector with P3HT:CdSe QDs = 1:5 active layer (TOP and pyridine ligands applied).

Table 1. Active layer thickness, relative permittivity, and mobility values of the detector with P3HT:CdSe QDs $=1: 5$ active layer (TOP and pyridine ligands applied).

\begin{tabular}{|c|c|c|c|c|c|}
\hline $\begin{array}{l}\text { Blending Ratio } \\
\text { (P3HT:CdSe) }\end{array}$ & Ligand & $\begin{array}{l}\text { Thickness } \\
\text { (nm) }\end{array}$ & Relative Permittivity $\left(\varepsilon_{r}\right)$ & $\mathrm{V}_{\mathrm{a}}(\mathrm{V})$ & $\begin{array}{c}\text { Mobility } \\
(\mu) \\
\left(\mathrm{cm}^{2} / \mathrm{V} \cdot \mathrm{s}\right)\end{array}$ \\
\hline \multirow{2}{*}{$1: 5$} & TOP & \multirow{2}{*}{50} & 3.2 & 0.5 & $8.33 \times 10^{-8}$ \\
\hline & Pyridine & & 7.3 & 1.22 & $1.03 \times 10^{-6}$ \\
\hline
\end{tabular}

In addition, the pyridine ligands had the effect of increasing the lowest unoccupied molecular orbital (LUMO) energy level, and they can affect the energy-band offset at the P3HT:CdSe QDs heterojunction. Figure 9 shows how after the pyridine ligand exchange, $\mathrm{J}_{\mathrm{SC}}, \mathrm{CCD}$, and the sensitivity of the detector with P3HT:CdSe QDs = 1:5 active layer increased. Compared to the detector fabricated with CdSe QDs TOP ligands, the sensitivity increased by about $75 \%$ after the pyridine ligand exchange.

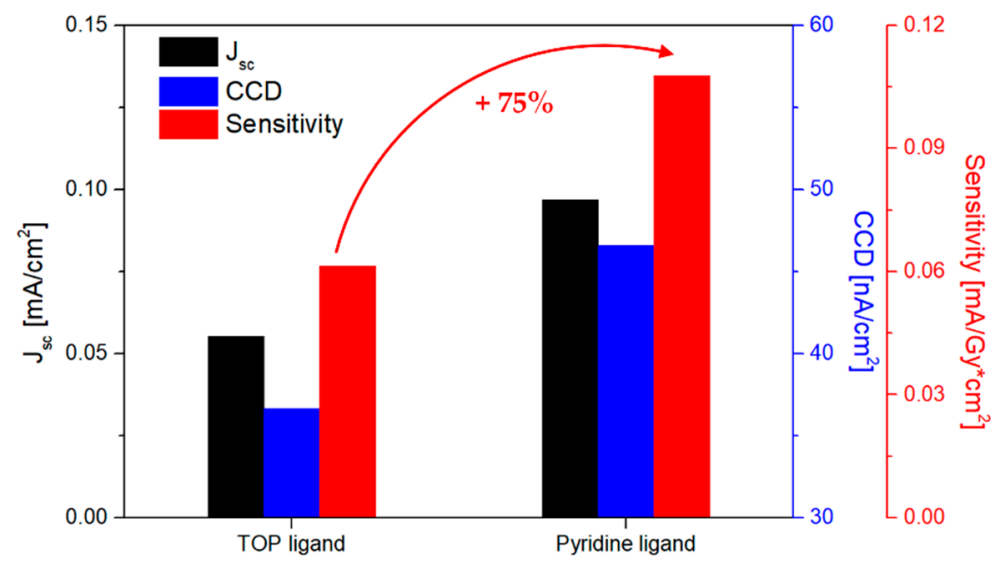

Figure 9. Changes in Jsc, CCD, and the sensitivity of the detector with P3HT:CdSe QDs = 1:5 active layer after ligand exchange.

In order to further improve the sensitivity of the proposed detector, we conducted an experiment on the process of forming the active layer. To control the thickness of the active layer, spin-coating conditions of $(700,700 / 1800,700 / 1500$, and 700/1200) rpm were changed. For example, the 700/1800 rpm 
condition meant that the spin coating was performed by dividing the active layer into two parts: 700 and $1800 \mathrm{rpm}$. In both cases, a P3HT:CdSe QD solution with ligand exchange was applied. In addition, pyridine treatment was inserted between the two spin-coating steps to evaluate its effect on active layer formation and detector performance. The solution used for double-coatings was a P3HT:CdSe QD active layer solution of the same conditions treated with pyridine. Figure 10 shows the detector with the $80 \mathrm{~nm}$ thick active layer formed by 700/1500 rpm double-coating and pyridine treatment revealed the highest $C C D$ and sensitivity. When the spin rate of the second spin-coating process was lowered to $1200 \mathrm{rpm}$, the thickness and surface roughness of the active layer simultaneously increased, while the CCD and sensitivity of the detector decreased. When the active layer was formed at one time under the spin-coating condition of $700 \mathrm{rpm}$, low CCD and sensitivity were observed because the thickness of the active layer was relatively thin, and the surface roughness was relatively high. In order to absorb visible light generated from the CsI (Tl) scintillator having a peak emission of $560 \mathrm{~nm}$, an organic active layer thickness of $80-90 \mathrm{~nm}$ is appropriate [26]. Additional pyridine treatment increased the CCD and sensitivity of the detector by preventing the aggregation of QDs and reducing the surface roughness of the active layer. When comparing the sensitivity of the detectors with the active layer formed under the same spin-coating conditions with or without pyridine treatment, the sensitivity of the pyridine-treated detector improved by about $9.36 \%$.

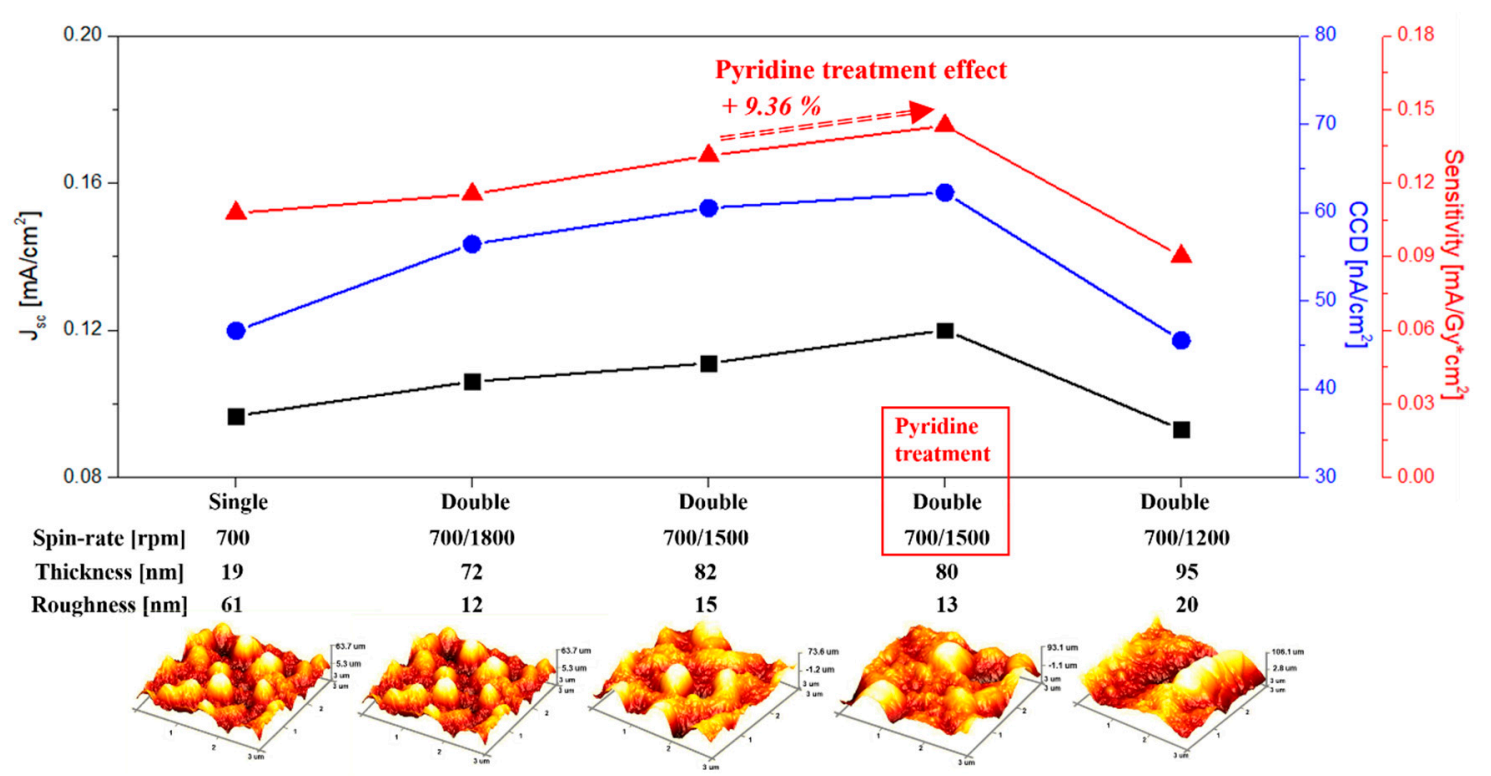

Figure 10. X-ray parameters (CCD and sensitivity), short-circuit current density $\left(\mathrm{J}_{\mathrm{sc}}\right)$, thickness of the active layer, and surface roughness of the active layer, depending on the spin-coating and pyridine treatment conditions.

\section{Conclusions}

This study investigated the characteristics of an indirect-type hybrid X-ray detector with a conjugated polymer P3HT and CdSe QD blended active layer. To improve detection sensitivity, the optimal blending ratio of P3HT:CdSe QDs, ligand exchange effect, and optimal process condition of the active layer were examined. First, the P3HT:CdSe QD thin films with different blending ratios of 1:2, 1:3,1:4, 1:5, and 1:6 were formed by a spin-coating process, and the absorbance of thin films was measured. As the content of CdSe QDs increased, the absorbance tended to increase due to its high extinction coefficient. After applying the same thin films as the active layer of the detector, the detector's performance was evaluated under X-ray irradiation. The detector with a P3HT:CdSe QDs $=1: 5$ blended active layer showed the highest $C C D$ and highest sensitivity. When the QD content in the active layer exceeded a certain level (in our case, 1:5 blending condition), the characteristics of the active layer became similar to those of the solid bulk. As a result, exciton formation and charge transfer 
were reduced, resulting in a deterioration of the detector's performance. At the optimized blending condition of P3HT:CdSe QDs = 1:5, a TOP ligand of QDs was replaced by a pyridine ligand, one of the short ligand molecules. The pyridine ligand replacement effectively assisted charge transport and reduced the QD aggregation, increasing the detection sensitivity of the detector by $75 \%$ after ligand exchange. To further improve the sensitivity of the proposed detector, the optimized process conditions of the active layer were studied. The sensitivity of the detector with the active layer of about $80 \mathrm{~nm}$ thickness formed by a double-coating method increased about $40 \%$, compared to the detector with the active layer about $60 \mathrm{~nm}$ thickness formed by a single-coating method. Due to the additional pyridine treatment between the two double-coating processes, the surface roughness of the active layer decreased, and the CCD and sensitivity subsequently increased. Finally, the hybrid detector with a P3HT:CdSe QD active layer under the optimized process conditions showed the highest CCD, $62.5 \mathrm{nA} / \mathrm{cm}^{2}$, and the highest sensitivity, $0.14 \mathrm{~mA} / \mathrm{Gy} \cdot \mathrm{cm}^{2}$.

Author Contributions: J.K. conceived this idea, and directed the experiment; S.K. and J.K. devised the experiment; S.K. and J.L. performed most of the detector fabrication and data collection; S.K. wrote the manuscript; J.L. and J.K. revised the manuscript. All authors have read and agreed to the published version of the manuscript.

Funding: This work was supported by the National Research Foundation of Korea (NRF) grant funded by the Korea government(MSIT) (No. 2017R1A2A2A05069821).

Conflicts of Interest: The authors declare no conflict of interest.

\section{References}

1. Paci, B.; Generosi, A.; Albertini, V.R.; Spyropoulos, G.D.; Stratakis, E.; Kymakis, E. Enhancement of photo/thermal stability of organic bulk heterojunction photovoltaic devices via gold nanoparticles doping of the active layer. Nanoscale 2012, 4, 7425-7459. [CrossRef] [PubMed]

2. Ikram, M.; Ali, S.; Murray, R.; Hussain, A.; Islah-u-din; Shah, S.I. Influence of fullerene derivative replacement with $\mathrm{TiO}_{2}$ nanoparticles in organic bulk heterojunction solar cells. Curr. Appl. Phys. 2015, 15, 48-54. [CrossRef]

3. Ikram, M.; Murray, R.; Hussain, A.; Ali, S.; Shah, S.I. Hybrid organic solar cells using both ZnO and PCBM as electron acceptor materials. Mater. Sci. Eng. B 2014, 189, 64-69. [CrossRef]

4. Roghabadi, F.A.; Kokabi, M.; Ahmadi, V.; Abaeiani, G. Structure optimization of P3HT: CdSe hybrid solar cell using optical analysis and electrochemical impedance spectroscopy. Thin Solid Films 2017, 621, 19-25. [CrossRef]

5. $\quad$ Plass, R.; Pelet, S.; Kruger, J.; Gratzel, M.; Bach, U. Quantum Dot Sensitization of Organic-Inorganic Hybrid Solar Cells. J. Phys. Chem. B 2002, 106, 7578-7580. [CrossRef]

6. Lee, Y.L.; Lo, Y.S. Highly efficient quantum-dot-sensitized solar cell based on co-sensitization of CdS/CdSe. Adv. Funct. Mater. 2009, 19, 604-609. [CrossRef]

7. Wang, J.; Ivan, M.S.; Pan, Z.; Zhao, K.; Zhang, H.; Feng, Y.; Yang, G.; Zhong, X.; Bisquert, J. Core/Shell Colloidal Quantum Dot Exciplex States for the Development of Highly Efficient Quantum-Dot-Sensitized Solar Cells. J. Am. Chem. Soc. 2013, 135, 15913-15922. [CrossRef]

8. Ciavatti, A.; Cramer, T.; Carroli, M.; Basirico, L.; Fuher, R.; De Leeuw, D.M.; Fraboni, B. Dynamics of direct X-ray detection processes in high- $\mathrm{Z}_{2} \mathrm{O}_{3}$ nanoparticles-loaded PFO polymer-based diodes. Appl. Phys. Lett. 2017, 111, 183301. [CrossRef]

9. Thirimanne, H.M.; Jayawardena, K.D.G.I.; Parnell, A.J.; Bandara, R.M.; Karalasingam, A.; Pani, S.; Huerdler, J.E.; Lidzey, D.G.; Tedde, S.F.; Nisbet, A.; et al. High sensitivity organic inorganic hybrid X-ray detectors with direct transduction and broadband response. Nat. Commun. 2018, 9, 2926. [CrossRef]

10. Kim, B.; Lee, J.; Kang, J. Improving the sensitivity of indirect-type organic X-ray detector by blending with CdSe quantum dots. J. Instrum. 2017, 12, C01009. [CrossRef]

11. Ankah, G.N.; Buchele, P.; Poulsen, K.; Rauch, T.; Tedde, S.F.; Gimmler, C.; Schmidt, O.; Kraus, T. PbS quantum dot based hybrid-organic photodetectors for X-ray sensing. Org. Electron. 2016, 33, 201-206. [CrossRef]

12. Buchele, P.; Richter, M.; Tedde, S.F.; Matt, G.J.; Ankah, G.N.; Fischer, R.; Biele, M.; Metzger, W.; Lilliu, S.; Bikondoa, O.; et al. X-ray imaging with scintillator-sensitized hybrid organic photodetectors. Nat. Photonics 2015, 9, 843. [CrossRef] 
13. Ciavatti, A.; Capria, E.; Alessandro, F.M.; Tromba, G.; Dreossi, D.; Sellin, P.J.; Cosseddu, P.; Bonfiglio, A.; Fraboni, B. Toward Low-Voltage and Bendable X-Ray Direct Detectors Based on Organic Semiconducting Single Crystals. Adv. Mater. 2015, 27, 7213-7220. [CrossRef] [PubMed]

14. Basirico, L.; Ciavatti, A.; Cramer, T.; Cosseddu, P.; Bonfiglio, A.; Fraboni, B. Direct X-ray photoconversion in flexible organic thin film devices operated below 1 V. Nat. Commun. 2016, 7, 13063. [CrossRef] [PubMed]

15. Lee, J.; Kang, J. Characteristics of a Flexible Radiation Detector Fabricated with Non-Fullerene Acceptor for an Indirect-type X-ray Imaging. J. Instrum. 2019, 14, C03008. [CrossRef]

16. Starkenburg, D.J.; Johns, P.M.; Baciak, J.E.; Nino, J.C.; Xue, J. Thin film organic photodetectors for indirect X-ray detection demonstrating low dose rate sensitivity at low voltage. J. Appl. Phys. 2017, 122, 225502. [CrossRef]

17. Lee, J.; Seon, H.; Kang, J. Comparative studies between photovoltaic and radiation parameters in indirect-type organic X-ray detector with a P3HT: PCBM active layer. Nanosci. Nanotechnol. Lett. 2017, 9, 1159-1164. [CrossRef]

18. Greaney, M.J.; Brutchey, R.L. Ligand engineering in hybrid polymer: Nanocrystal solar cells. Mater. Today 2015, 18, 31-38. [CrossRef]

19. Zilner, E.; Fengler, S.; Niyamakom, P.; Rauscher, F.; Kogler, K.; Dittrich, T. Role of ligand exchange at CdSe quantum dot layers for charge separation. J. Phys. Chem. C 2012, 116, 16747-16754. [CrossRef]

20. Lokteva, I.; Radychev, N.; Witt, F.; Borchert, H.; Parisi, J.; Joanna, K.O. Surface treatment of CdSe nanoparticles for application in hybrid solar cells: The effect of multiple ligand exchange with pyridine. J. Phys. Chem. C 2010, 114, 12784-12791. [CrossRef]

21. Zhu, L.; Richardson, B.J.; Yu, Q. Inverted hybrid CdSe-Polymer solar cells adopting PEDOT: PSS/MoO 3 as dual hole transport layers. Phys. Chem. Chem. Phys. 2016, 18, 3463-3471. [CrossRef] [PubMed]

22. Abargues, R.; Navarro, J.; Rodriguez-Canto, J.P.; Maulu, A.; Sanchez-Royo, F.J.; Martinez-Pastor, P.J. Enhancing the photocatalytic properties of PbS QD solids: The ligand exchange approach. Nanoscale 2019, 11, 1978-1987. [CrossRef] [PubMed]

23. Kraner, S. Measurement of Charge Carrier Mobility and Charge Carrier Concentration of Organic Photovoltaic Diodes under In Situ Light Soaking Conditions and Varying Temperatures. Master's Thesis, Johannes Kepler University, Linz, Austria, 2011.

24. Takeshita, S. Modeling of Space-Charge-Limited Current Injection Incorporating an Advanced Model of the Poole-Frenkel Effect. Master's Thesis, Clemson University, Clemson, SC, USA, 2008.

25. Sareni, B.; Krahenbuhl, L.; Beroual, A. Effective dielectric constant of random composite materials. J. Appl. Phys. 1997, 81, 2375-2383. [CrossRef]

26. Seon, H.; Ban, D.; Kang, J. A study on the characteristics of indirect-type organic detector using p-type polycarbazole copolymer for X-ray. J. Instrum. 2018, 13, C01031. [CrossRef] 